\title{
Pion $p_{T}$ Spectra in $p+p$ Collisions as a Function of $\sqrt{s}$ and Event Multiplicity
}

\author{
Priyanka Sett ${ }^{1,2}$ and Prashant Shukla ${ }^{1,2}$ \\ ${ }^{1}$ Nuclear Physics Division, Bhabha Atomic Research Center, Trombay, Mumbai 400 085, India \\ ${ }^{2}$ Homi Bhabha National Institute, Anushaktinagar, Mumbai 400094, India \\ Correspondence should be addressed to Priyanka Sett; sett.priyanka@gmail.com
}

Received 27 June 2014; Accepted 15 August 2014; Published 2 September 2014

Academic Editor: Edward Sarkisyan-Grinbaum

Copyright (C) 2014 P. Sett and P. Shukla. This is an open access article distributed under the Creative Commons Attribution License, which permits unrestricted use, distribution, and reproduction in any medium, provided the original work is properly cited. The publication of this article was funded by $\mathrm{SCOAP}^{3}$.

\begin{abstract}
We study the charged pion transverse momentum $\left(p_{T}\right)$ spectra in $p+p$ collisions as a function of collision energy $\sqrt{s}$ and event multiplicity using Tsallis distribution. This study gives an insight of the pion production process in $p+p$ collisions. The study covers pion spectra measured in $p+p$ collisions at SPS energies $(6.27-17.27 \mathrm{GeV})$, RHIC energies $(62.4 \mathrm{GeV}$ and $200 \mathrm{GeV})$, and LHC energies $(900 \mathrm{GeV}, 2.76 \mathrm{TeV}$, and $7 \mathrm{TeV})$. The Tsallis parameters have been obtained and parameterized as a function of $\sqrt{s}$. The study suggests that as we move to higher energy more and more hard processes contribute to the spectra. We also study the charged pion spectra for different event multiplicities in $p+p$ collisions for LHC energies using Tsallis distribution. The variation of the Tsallis parameters as a function of event multiplicity has been obtained and their behavior is found to be independent of collision energy.
\end{abstract}

\section{Introduction}

The particle spectra measured in hadronic collisions are of utmost interest because of their fundamental nature and simplicity, which allow verifying pQCD $[1,2]$ calculations and also help to make comprehensive phenomenological studies. The ratios of the particle yields obtained from the measured spectra allow getting the chemical freeze-out conditions, whereas the spectra themselves reflect the conditions at the kinetic freeze-out. The particle spectra provide useful information about the collision dynamics. The low $p_{T}$ region of the spectrum corresponds to the particles originating from low momentum transfer and multiscattering processes (nonperturbative QCD), whereas the high $p_{T}$ region comes from the hard-parton-scattering (pQCD) among the initial partons. The transition of this nonperturbative to perturbative dynamics has no sharp boundary, though one can have an estimate from the " $x_{T}$-scaling" $[3]$. Extensive $[4,5]$ and nonextensive [6-10] statistical approaches have been used to characterize particle spectra in terms of thermodynamic variables. Extensive statistics assume thermal and chemical equilibrium of the system at hadronic phase which lead to an exponential distribution of the particle spectra. In experiments, the particle spectra show a power-law behavior at high $p_{T}$. This behavior is reproduced by the nonextensive approach with an additional parameter. In recent times, the Tsallis [6] statistical approach is widely used to describe the particle spectra obtained in high-energy collisions with only two parameters, the temperature $T$ and $q$, known as nonextensivity parameter which is a measure of temperature fluctuations or degree of nonequilibrium in the system.

The Tsallis distribution gives an excellent description of $p_{T}$ spectra of all identified mesons measured in $p+p$ collisions at $\sqrt{s}=200 \mathrm{GeV}$ [11]. In a recent work [12, 13], the Tsallis distribution has been used to describe the $p_{T}$ spectra of identified charged hadrons measured in $p+p$ collisions at RHIC and at LHC energies. Such an approach has also been applied to the inclusive charged hadron $p+p$ data in recent publications $[14,15]$. It has been shown in $[12,16]$ that the functional form of the Tsallis distribution with thermodynamic origin is of the same form as the QCDinspired Hagedorn formula [17, 18]. This could be the reason 
of success of Tsallis distribution in $p+p$ collisions which is a power law typical of QCD hard scatterings. The hardness of the spectra is thus related to $q$ and the parameter $T$ governs the contribution from soft collisions.

Using the Tsallis phenomenological function, we review and study the charged pion spectra in $p+p$ collisions in a large energy regime, spanning from SPS [19] $(6.27 \mathrm{GeV}-$ $17.27 \mathrm{GeV})$ and RHIC [20] (62.4 and $200 \mathrm{GeV})$ to LHC [21] $(900 \mathrm{GeV}, 2.76 \mathrm{TeV}$ and $7 \mathrm{TeV})$ energies. The object of the present work is to study the behaviour of the Tsallis parameters as a function of collision energy. We also study the charged pion spectra for different event multiplicities in $p+p$ collisions for LHC energies. Among all hadrons, pions are chosen because of their abundance in collisions, simple quark structure, and availability of the data at different energies.

\section{Formalism}

The transverse momentum spectra of hadrons, obtained from different fixed and collider experiments, have shown that the high $p_{T}$ region of the spectra can be described successfully by the power law,

$$
E \frac{d^{3} N}{d p^{3}}=C_{P} p_{T}^{-n}
$$

where $C_{P}$ is the normalization constant and $n$ is the power which determines the shape of the spectra at high $p_{T}$. However, the low $p_{T}$ region of the particle spectra shows an exponential shape and can be described by the BoltzmannGibbs [22, 23] statistical approach,

$$
E \frac{d^{3} N}{d p^{3}}=C_{B} e^{-E / T}
$$

where $C_{B}$ is the normalization constant, $E$ is the particle energy, and $T$ is the temperature of the system.

In the early 80s, Hagedorn [17] proposed a phenomenological function which describes the particle spectra for both the higher and lower $p_{T}$ regions:

$$
E \frac{d^{3} N}{d p^{3}}=A\left(1+\frac{p_{T}}{p_{0}}\right)^{-n}
$$

where $A, p_{0}$, and $n$ are the fit parameters. The above equation describes an exponential behavior for low $p_{T}$ and a power-law behavior for high $p_{T}$. Consider

$$
\begin{aligned}
\left(1+\frac{p_{T}}{p_{0}}\right)^{-n} & \simeq \exp \left(\frac{-n p_{T}}{p_{0}}\right), \quad \text { for } p_{T} \longrightarrow 0 \\
& \simeq\left(\frac{p_{0}}{p_{T}}\right)^{n}, \quad \text { for } p_{T} \longrightarrow \infty
\end{aligned}
$$

The parameter $n$ in this equation is often related to the "power" in the "QCD-inspired" quark interchange model [18].

In the late $80 \mathrm{~s}$, Tsallis [6] introduced the idea of the nonextensive statistics in place of thermal Boltzmann-Gibbs statistics. This approach includes a parameter $q$, known as nonextensive parameter which quantifies the temperature fluctuation [24] in the system as $q-1=\operatorname{Var}(1 / T) /\langle T\rangle^{2}$. The nonextensive statistics assume Boltzmann-Gibbs form in the limit $q \rightarrow 1$. In Tsallis approach, the Boltzmann-Gibbs distribution takes the form

$$
E \frac{d^{3} N}{d p^{3}}=C_{q}\left(1+(q-1) \frac{E}{T}\right)^{-1 /(q-1)},
$$

where $C_{q}$ is the normalization factor. One can use the relation $E=m_{T}$ at mid-rapidity and $n=1 /(q-1)$ in (5) to obtain

$$
E \frac{d^{3} N}{d p^{3}}=C_{n}\left(1+\frac{m_{T}}{n T}\right)^{-n}
$$

where $C_{n}$ is the normalization factor. Equation (6) can be rewritten as

$$
\frac{1}{2 \pi p_{T}} \frac{d^{2} N}{d p_{T} d y}=C_{n}\left(1+\frac{m_{T}}{n T}\right)^{-n},
$$

The value of $C_{n}$ can be obtained by integrating (7) over momentum space:

$$
C_{n}=\frac{d N / d y}{\int_{0}^{\infty}\left(1+\left(m_{T} / n T\right)\right)^{-n} 2 \pi p_{T} d p_{T}} .
$$

Here the quantity $d N / d y$ is the $p_{T}$ integrated yield. Equation (6) with the normalization constant takes the form [11]

$$
\begin{aligned}
E \frac{d^{3} N}{d p^{3}}= & \frac{1}{2 \pi} \frac{d N}{d y} \frac{(n-1)(n-2)}{(n T+m(n-1))(n T+m)} \\
& \times\left(\frac{n T+m_{T}}{n T+m}\right)^{-n} .
\end{aligned}
$$

Larger values of $q$ correspond to smaller values of $n$ which imply dominant hard QCD point-like scattering. Both $n$ and $q$ have been interchangeably used in Tsallis distribution $[7,11,20,25,26]$. The Tsallis interpretation of parameters $T$ as temperature and $q$ as nonextensivity parameter is more suited for heavy ion collisions while for $p+p$ collisions Hagedorn interpretation in terms of power $n$ and a parameter $T=$ $p_{0} / n$ which controls soft physics processes is more meaningful. Phenomenological studies suggest that, for quark-quark point scattering, $n \sim 4[27,28]$ and when multiple scattering centers are involved, $n$ grows larger.

There are many other forms of (9), which are used often to describe particle spectra in literature; see, for example, [7, 20, 25, 26, 29-31].

\section{Results and Discussions}

All the studies are performed with (9) and the fit parameters $n, T$, and $d N / d y$ are obtained. The different experiments, energies, rapidity ranges, and particles used in the analysis are summarized in Table 1. For SPS energies only available data is for $\pi^{-}$measured by NA61 Collaboration [19]; for RHIC and LHC energies we use $\left(\pi^{+}+\pi^{-}\right) / 2$. All the data used 
TABLE 1: The center of mass energy and rapidity of the data used for the study.

\begin{tabular}{lccc}
\hline Experiments & $\begin{array}{c}\text { Center of mass } \\
\text { energy }(\mathrm{GeV})\end{array}$ & Rapidity & $\begin{array}{c}\text { Particles } \\
\text { studied }\end{array}$ \\
\hline SPS & $6.27,7.74,8.76,12.32,17.27$ & $0.0-0.2$ & $\pi^{-}$ \\
RHIC & $62.4,200$ & $|y|<0.35$ & $\pi^{+}, \pi^{-}$ \\
LHC & $900,2760,700$ & $|y|<1.0$ & $\pi^{+}, \pi^{-}$ \\
\hline
\end{tabular}

TABLE 2: Values of the $\chi^{2} / \mathrm{NDF}$ for Tsallis fits of pion spectra at different $\sqrt{s}$.

\begin{tabular}{lc}
\hline$\sqrt{s}$ & $\chi^{2} / \mathrm{NDF}$ \\
\hline $6.27 \mathrm{GeV}$ & $6.31 / 12$ \\
$7.74 \mathrm{GeV}$ & $5.80 / 12$ \\
$8.76 \mathrm{GeV}$ & $10.68 / 12$ \\
$12.32 \mathrm{GeV}$ & $9.25 / 12$ \\
$17.27 \mathrm{GeV}$ & $2.65 / 12$ \\
$62 \mathrm{GeV}$ & $0.74 / 11$ \\
$200 \mathrm{GeV}$ & $0.48 / 11$ \\
$900 \mathrm{GeV}$ & $24.33 / 19$ \\
$2.76 \mathrm{TeV}$ & $5.59 / 19$ \\
$7.00 \mathrm{TeV}$ & $13.11 / 19$ \\
\hline
\end{tabular}

are measured in mid-rapidity and are given for unit rapidity. The difference in rapidity range is not expected to affect the behaviour of the spectra. CMS experiment presented [21] transverse momentum spectra for different events classified on the basis of number of true tracks here referred to as track multiplicity of event or simply multiplicity. Each multiplicity class is represented by average number of tracks $\left(\left\langle N_{\text {tracks }}\right\rangle\right)$.

3.1. Tsallis Parameters as a Function of $\sqrt{s}$ in $p+p$ System. In this analysis all the Tsallis parameters are obtained for charged pion spectra as a function of $\sqrt{s}$ in $p+p$ system for SPS [19], RHIC [20], and LHC [21] energies. Similar study is available in [12] using RHIC and LHC data and in [13] for SPS and LHC data.

The pion $p_{T}$ spectra measured in $p+p$ collisions at different $\sqrt{s}$ are shown in Figure 1 along with with Tsallis fits (9) shown by solid lines. The spectra are scaled by arbitrary factors (given in figure) for visual clarity. In case of RHIC data, we restrict the $p_{T}$ range to $1.7 \mathrm{GeV} / \mathrm{c}^{2}$ to have similar $p_{T}$ range at all energies. It can be noticed that the spectra become harder with increase in $\sqrt{s}$ which is depictive of occurrence of harder scatterings at higher collision energy. The $\chi^{2}$ per degree of freedom $\chi^{2} / \mathrm{NDF}$ values for all the fits are given in Table 2 . The $\chi^{2} / \mathrm{NDF}$ values are $\lesssim 1$, which is indicative of good fit quality.

The parameters $n$ and $T$ obtained from this analysis are shown in Figures 2 and 3, respectively, as a function of $\sqrt{s}$. The variation of $d N / d y$ as a function of $\sqrt{s}$ is shown in Figure 4 . The parameter $n$ decreases with increasing $\sqrt{s}$ and starts saturating at LHC energies. The value of $T$ also reduces

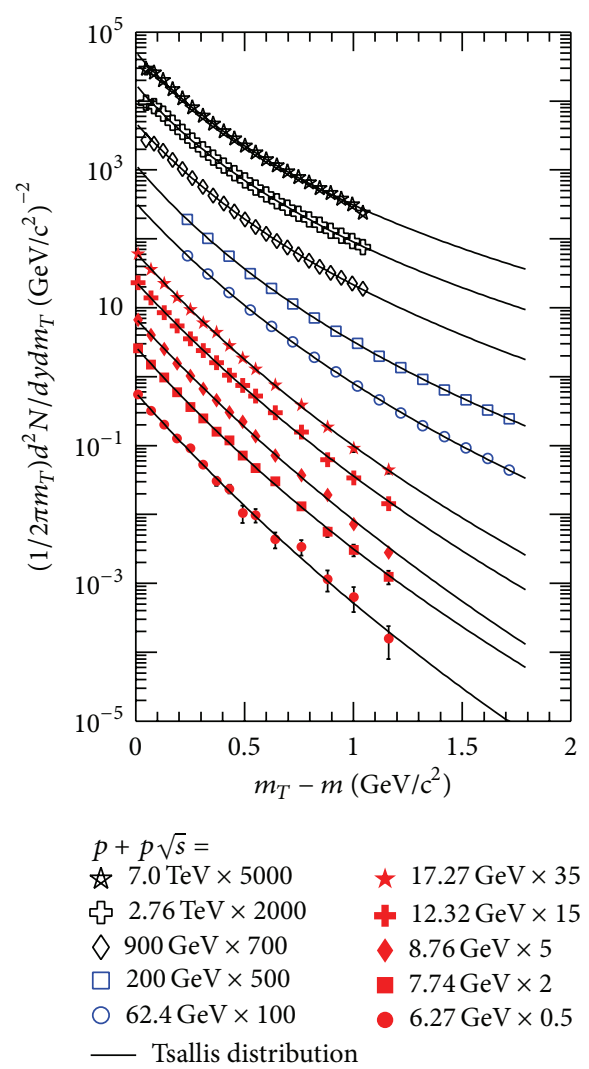

FIGURE 1: The invariant yield spectra of charged pions as a function of $\left(m_{T}-m\right)$ for SPS [19] energies $6.27 \mathrm{GeV}, 7.74 \mathrm{GeV}, 8.76 \mathrm{GeV}$, $12.32 \mathrm{GeV}$, and $17.27 \mathrm{GeV}$, RHIC [20] energies $62.4 \mathrm{GeV}$ and $200 \mathrm{GeV}$, and LHC [21] energies $900 \mathrm{GeV}, 2.76 \mathrm{TeV}$, and $7 \mathrm{TeV}$. The solid lines are the Tsallis function (9). The negative pion yields are plotted for SPS energies and for all other energies; average yield for positive and negative pion is plotted.

slowly from SPS energies to LHC energies. The integrated yield $d N / d y$ increases 10 times when going from SPS to highest LHC energy.

Larger value of $n$ (also larger value of $T$ ) suggests that the spectra have contribution from processes involving small momentum transfer arising due to the rescattering, recombination of partons, fragmentation from strings, and so forth, whereas smaller values of $n$ are indicative of harder processes being involved in particle production. Thus the spectra at SPS energies have large softer contribution and as the collision energy increases more and more contribution from hard processes is added.

All the three parameters can be parametrized by a function of type

$$
f(\sqrt{s})=\left(a+(\sqrt{s})^{-\alpha}\right)^{b}
$$

Here $a=1.33 \pm 0.08, \alpha=0.22 \pm 0.06$, and $b=4.36 \pm 0.24$ for $n(\sqrt{s}), a=2.63 \pm 0.62, \alpha=0.04 \pm 0.02$, and $b=3.76 \pm$ 0.49 for $T(\sqrt{s})$, and $a=0.65 \pm 0.01, \alpha=0.22 \pm 0.01$, and $b=-4.78 \pm 0.03$ for $d N(\sqrt{s}) / d y$. Using the parameterizations for $n$ by (10) we get $n \sim 3.46$ in the limit $\sqrt{s} \rightarrow \infty$. The 


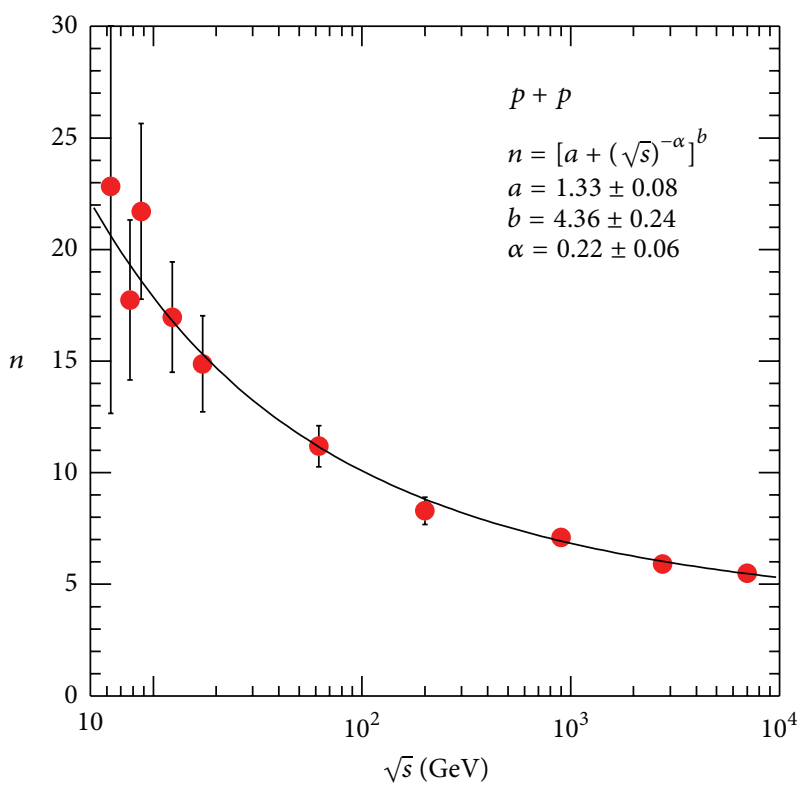

FIGURE 2: The variation of the Tsallis parameter $n$ for charged pions as a function of $\sqrt{s}$. The solid curve represents the parameterization $\left(a+(\sqrt{s})^{-\alpha}\right)^{b}$.

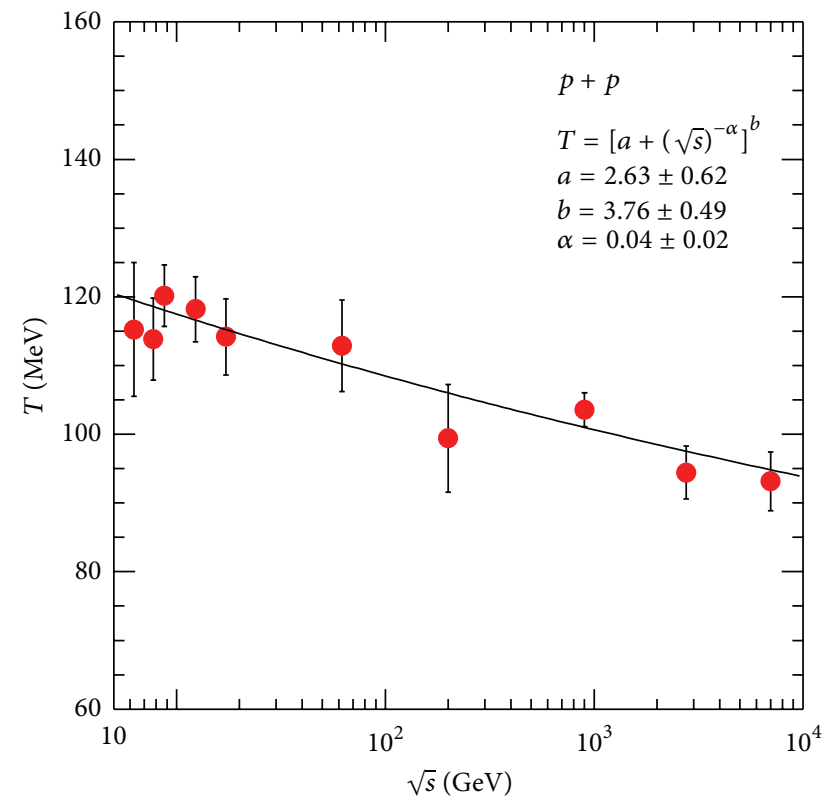

FIgURE 3: The variation of the Tsallis parameter $T$ for charged pions as a function of $\sqrt{s}$. The solid curve represents the parameterization $\left(a+(\sqrt{s})^{-\alpha}\right)^{b}$.

extrapolated values for $n, T$ and $d N / d y$ for $\sqrt{s}=14 \mathrm{TeV}$ are $n \sim 5.09, T \sim 90.33 \mathrm{MeV}$, and $d N / d y \sim 3.44$.

\subsection{Tsallis Parameters as a Function of Multiplicity $\left(\left\langle N_{\text {tracks }}\right\rangle\right)$} for LHC Energies. The Tsallis parameters for charged pion spectra are studied as a function of event multiplicity for different LHC energies $900 \mathrm{GeV}$ and 2.76 and $7 \mathrm{TeV}$. The event

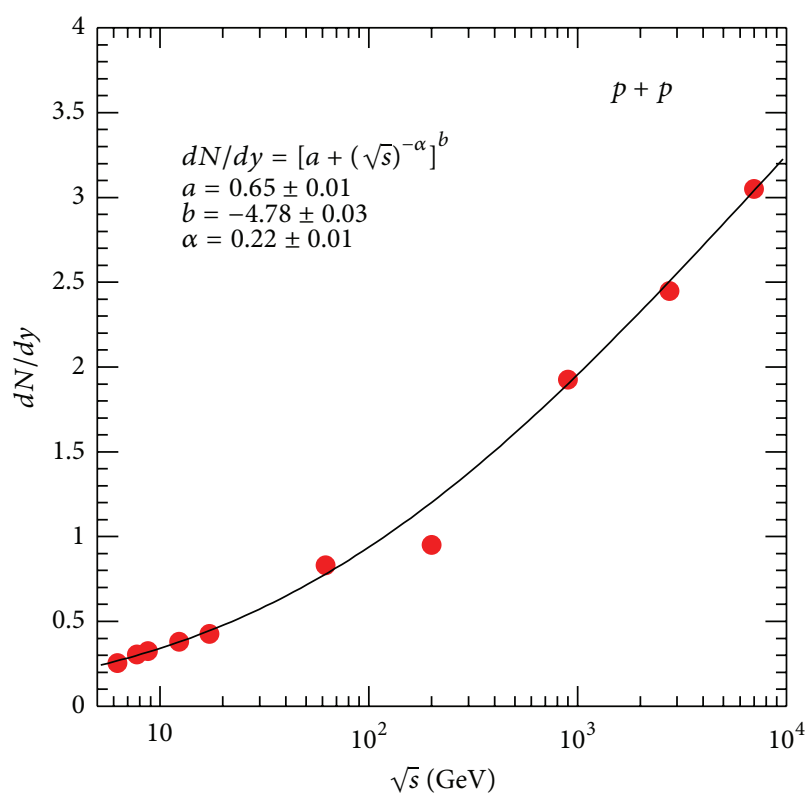

FIGURE 4: The variation of the integrated yield $d N / d y$ for charged pions as a function of $\sqrt{s}$. The solid curve represents the parameterization $\left(a+(\sqrt{s})^{-\alpha}\right)^{b}$.

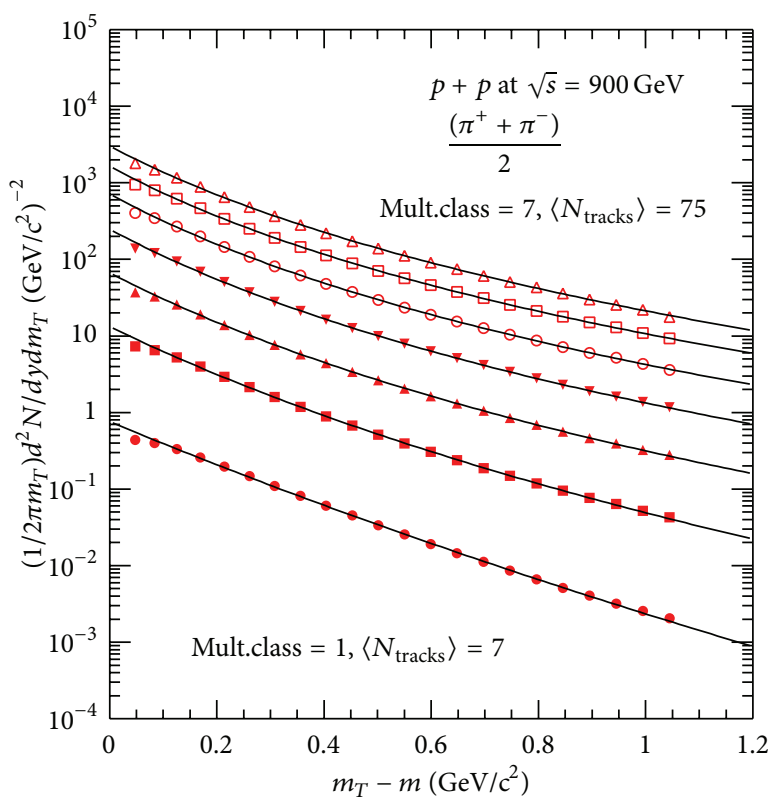

FIgURE 5: The invariant yield spectra of $\left(\pi^{+}+\pi^{-}\right) / 2$ [21], as a function of $m_{T}-m$ for $p+p$ collisions at $\sqrt{s}=900 \mathrm{GeV}$. The yields are shown for $\left\langle N_{\text {tracks }}\right\rangle 7,16,28,40,52,63$, and 75 . The spectra are scaled up for clarity by a factor of $6^{i}$, where $i=0,1,2,3,4,5$, and 6 . The solid lines show the Tsallis fits.

multiplicity data was also studied in a recent work [10] but our analysis and interpretations are different.

The invariant yield spectra corresponding to different multiplicities are fitted with (9) and are shown by the solid black lines in Figure 5 for $900 \mathrm{GeV}$, in Figure 6 for 2.76 TeV, and in Figure 7 for $7 \mathrm{TeV}$ center of mass energy. The 


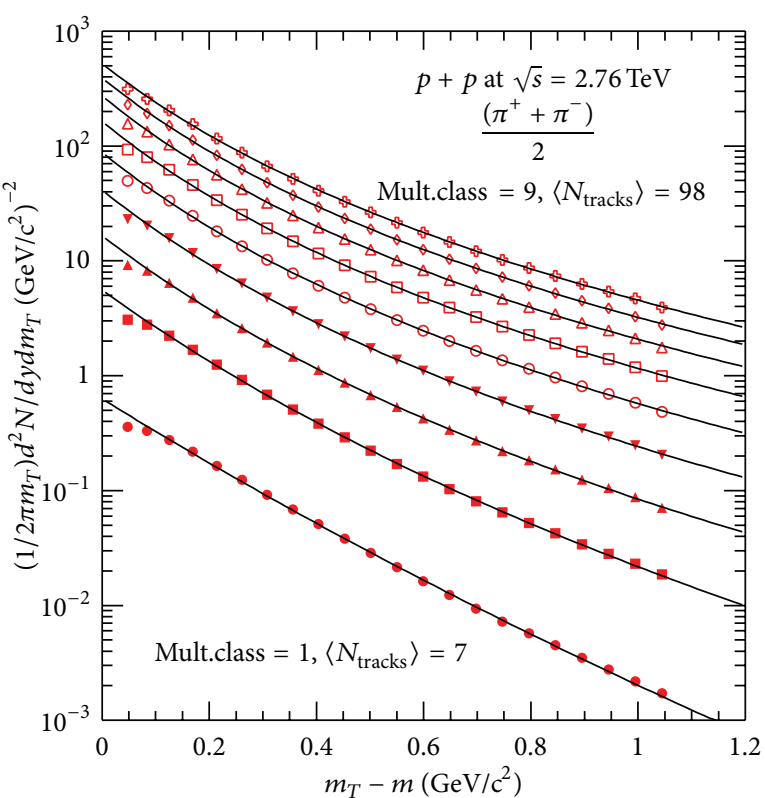

FIGURE 6: The invariant yield spectra of $\left(\pi^{+}+\pi^{-}\right) / 2$ [21], as a function of $m_{T}-m$ for $p+p$ collisions at $\sqrt{s}=2.76 \mathrm{TeV}$. The yields are shown for $\left\langle N_{\text {tracks }}\right\rangle 7,16,28,40,52,63,75,86$, and 98 . The spectra are scaled up for clarity by a factor of $3^{i}$, where $i=0,1,2,3,4,5,6,7$, and 8 . The solid lines show the Tsallis fits.

TABLE 3: Values of the $\chi^{2} / \mathrm{NDF}$ for the Tsallis fits in different event multiplicities.

\begin{tabular}{lccc}
\hline$\left\langle N_{\text {tracks }}\right\rangle$ & \multicolumn{3}{c}{$\chi^{2} / \mathrm{NDF}$ values for } \\
\hline 7 & $900 \mathrm{GeV}$ & $2.76 \mathrm{TeV}$ & $7.0 \mathrm{TeV}$ \\
16 & $48.89 / 18$ & $57.66 / 18$ & $48.10 / 18$ \\
28 & $25.21 / 18$ & $26.38 / 18$ & $27.76 / 18$ \\
40 & $9.97 / 18$ & $11.47 / 18$ & $22.83 / 18$ \\
52 & $7.20 / 18$ & $6.91 / 18$ & $19.25 / 18$ \\
63 & $8.00 / 18$ & $6.08 / 18$ & $22.52 / 18$ \\
75 & $9.18 / 18$ & $6.44 / 18$ & $26.30 / 18$ \\
86 & $15.01 / 18$ & $9.05 / 18$ & $21.30 / 18$ \\
98 & & $8.16 / 18$ & $19.24 / 18$ \\
109 & & $11.91 / 18$ & $23.59 / 18$ \\
120 & & & $20.82 / 18$ \\
131 & & & $16.85 / 18$ \\
\hline
\end{tabular}

spectra are scaled up for distinctness. The Tsallis distribution describes all the spectra well, shown by the $\chi^{2} / \mathrm{NDF}$ values given in Table 3 . The $\chi^{2} / \mathrm{NDF}$ values are little higher for some of the lower multiplicities due to the deviation of first data point in $p_{T}$ spectra with the curve.

The parameters $n$ and $T$ obtained from the fits are shown in Figures 8 and 9, respectively, as a function of $\left\langle N_{\text {tracks }}\right\rangle$. The circles, squares, and triangles correspond to the parameter values obtained from data at $900 \mathrm{GeV}, 2.76 \mathrm{TeV}$, and $7 \mathrm{TeV}$, respectively. It is seen that both the parameters $n$ and $T$ decrease rapidly and then start saturating with the increase of $\left\langle N_{\text {tracks }}\right\rangle$ for all three energies. This variation (of $n$ and $T$ )

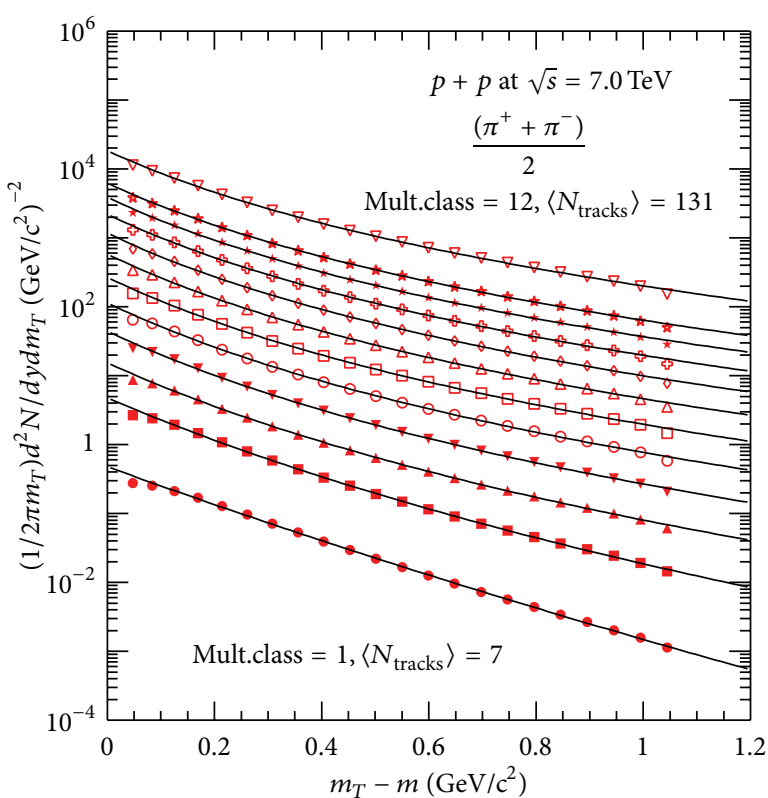

FIGURE 7: The invariant yield spectra of $\left(\pi^{+}+\pi^{-}\right) / 2$ [21], as a function of $m_{T}-m$ for $p+p$ collisions at $\sqrt{s}=7.00 \mathrm{TeV}$. The yields are shown for $\left\langle N_{\text {tracks }}\right\rangle 7,16,28,40,52,63,75,86,98,109,120$, and 131. The spectra are scaled up for clarity by a factor of $3^{i}$, where $i=0,1,2,3,4,5,6,7,8,9,10$, and 11 . The solid lines show the Tsallis fits.

is very similar to the variation which we find as a function of $\sqrt{s}$. It means that events with higher multiplicity have larger contribution from hard processes. The value of $n$ for high multiplicity events at $7 \mathrm{TeV}$ is $\sim 4$ which is depictive of production from point quark-quark scattering. The variation of $n$ and $T$ as a function of $\left\langle N_{\text {tracks }}\right\rangle$ can be described by the same curve given in the figure for all three energies and are parameterized by

$$
f\left(\left\langle N_{\text {tracks }}\right\rangle\right)=\left(a+\left(\left\langle N_{\text {tracks }}\right\rangle\right)^{-\alpha}\right)^{b} .
$$

Here $a=1.13 \pm 0.01, \alpha=0.81 \pm 0.04$, and $b=10.32 \pm 0.76$ for $n\left(\left\langle N_{\text {tracks }}\right\rangle\right)$ and $a=2.20 \pm 0.06, \alpha=0.56 \pm 0.08$, and $b=5.33 \pm 0.23$ for $T\left(\left\langle N_{\text {tracks }}\right\rangle\right)$.

The $p_{T}$ integrated pion yield distribution in different multiplicity classes is shown in Figure 10 for the three LHC energies. The total $p_{T}$ integrated pion yield for each energy can be obtained by integrating the above distributions over all multiplicity classes. It is noticed that as the energy increases more and more high mutliplicity events are added in the sample with mean of the distribution shifting towards higher $\left\langle N_{\text {tracks }}\right\rangle$.

\section{Conclusion}

This work presented the study of the transverse momentum spectra of the charged pions for different collisional energies and also for different event multiplicities (at LHC energies) using Tsallis distribution. The Tsallis parameter $n$ decreases with increasing $\sqrt{s}$ and starts saturating at LHC energies. 


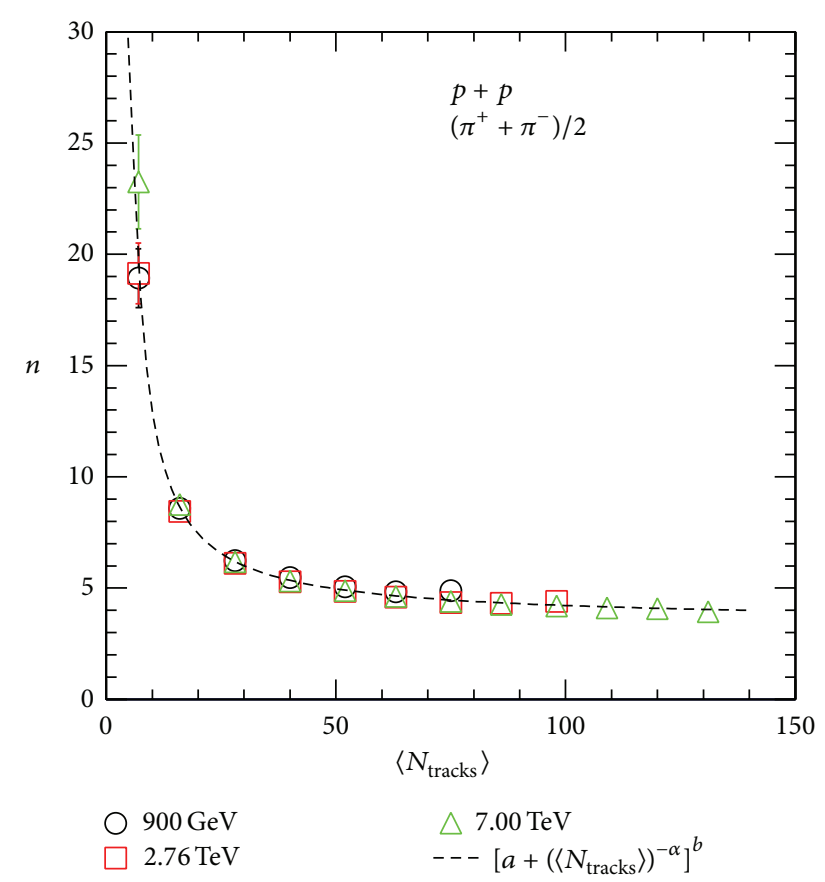

FIgURE 8: The variation of the Tsallis parameter $n$ for charged pions as a function of $\left\langle N_{\text {tracks }}\right\rangle$. The variation is shown for $900 \mathrm{GeV}$ by black circles, $2.76 \mathrm{TeV}$ by red squares, and $7.00 \mathrm{TeV}$ by green triangles. The dashed curve represents the parameterization $\left(a+\left(\left\langle N_{\text {tracks }}\right\rangle\right)^{-\alpha}\right)^{b}$.

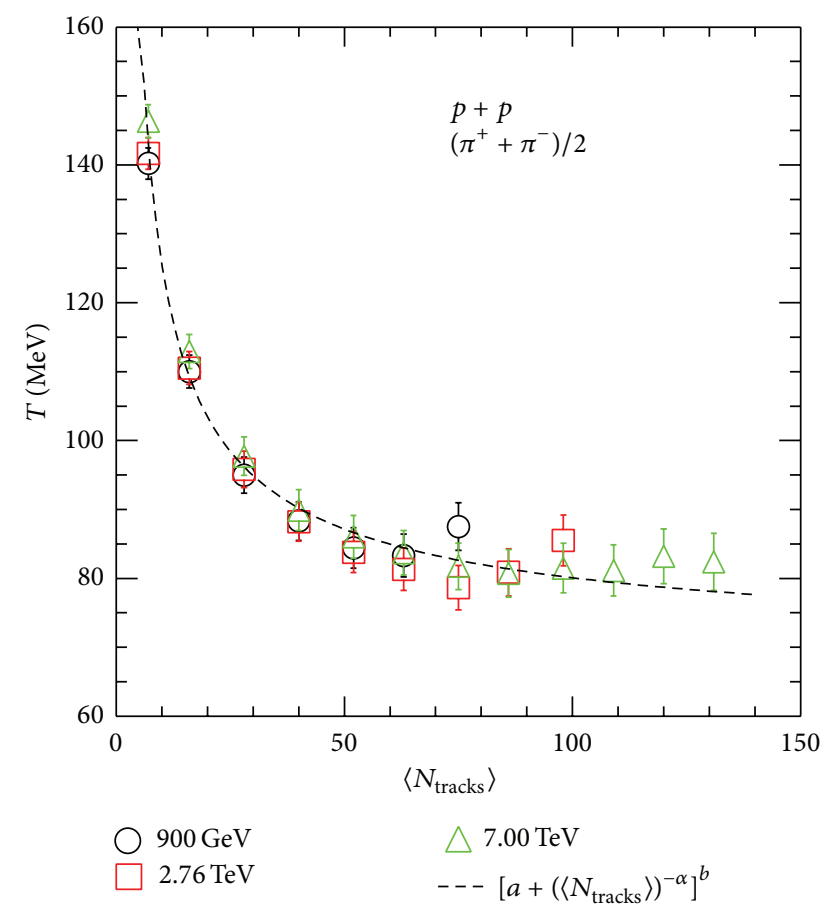

FIGURE 9: The variation of the Tsallis parameter $T$ for charged pions as a function of $\left\langle N_{\text {tracks }}\right\rangle$. The variation is shown for $900 \mathrm{GeV}$ by black circles, $2.76 \mathrm{TeV}$ by red squares, and $7.00 \mathrm{TeV}$ by green triangles. The dashed curve represents the parameterization $\left(a+\left(\left\langle N_{\text {tracks }}\right\rangle\right)^{-\alpha}\right)^{b}$.

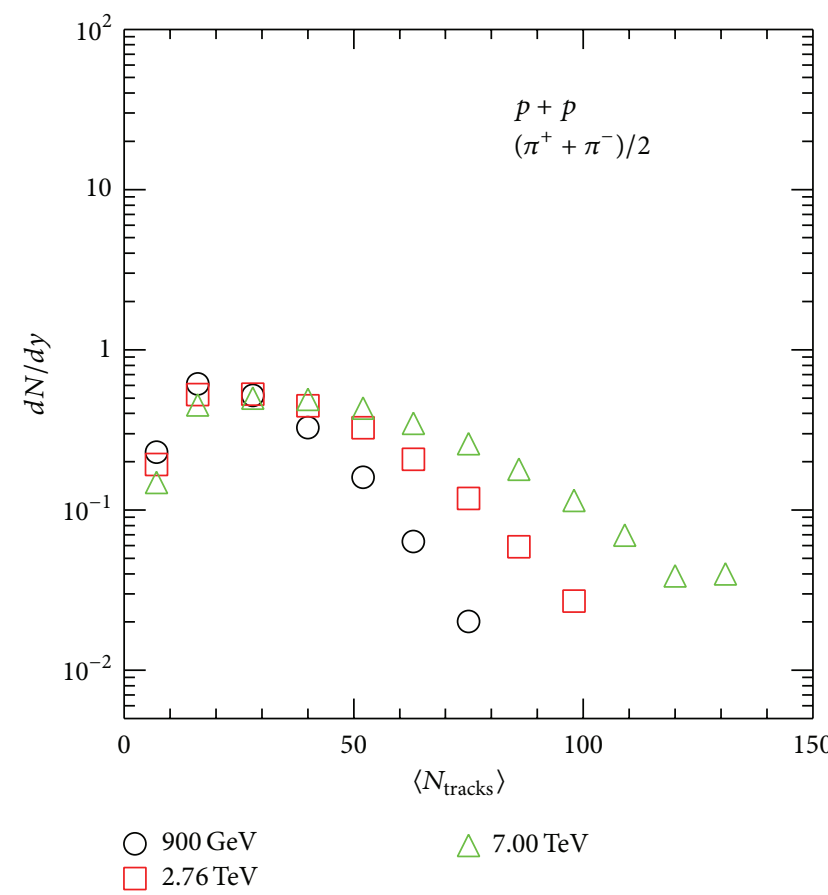

FIGURE 10: The variation of the integrated yield $d N / d y$ for charged pions as a function of $\left\langle N_{\text {tracks }}\right\rangle$. The variation is shown for $900 \mathrm{GeV}$ by black circles, $2.76 \mathrm{TeV}$ by red squares, and $7.00 \mathrm{TeV}$ by green triangles.

The value of $T$ also reduces slowly from SPS energies to LHC energies. It means that the spectra at SPS energies have large softer contribution and as the collision energy increases more and more contribution from hard processes is added. The $p_{T}$ integrated pion yield increases with increasing $\sqrt{s}$ and becomes 10 times when going from SPS to highest LHC energy. The Tsallis parameters are also obtained as a function of event multiplicity for all three LHC energies which can be described by the same curve. The variation of $n$ and $T$ as a function of multiplicity is very similar to the variation which we find as a function of $\sqrt{s}$. It means that events with higher multiplicity have larger contribution from hard processes. The value of $n$ for high multiplicity events at $7 \mathrm{TeV}$ is $\sim 4$ which is depictive of production from point quarkquark scattering. The $p_{T}$ integrated pion yield distribution for the three LHC energies shows that as the energy increases, more and more high mutliplicity events are added in the sample with mean of the distribution shifting towards higher multiplicity.

\section{Conflict of Interests}

The authors declare that there is no conflict of interests regarding the publication of this paper.

\section{References}

[1] G. Sterman, J. Smith, J. C. Collins (CTEQ Collaboration) et al., "Handbook of perturbative QCD," Reviews of Modern Physics, vol. 67, article 157, 1995. 
[2] J. F. Owens, E. Reya, and M. Glück, "Detailed quantumchromodynamic predictions for high- p $_{T}$ processes," Physical Review D, vol. 18, p. 1501, 1978.

[3] R. Blankenbecler, S. J. Brodsky, and J. F. Gunion, "Inclusive processes at high transverse momentum," Physics Letters B, vol. 42, no. 4, pp. 461-465, 1972.

[4] E. Fermi, "High energy nuclear events," Progress of Theoretical Physics, vol. 5, no. 4, pp. 570-583, 1950.

[5] A. Andronic, P. Braun-Munzinger, and J. Stachel, "Hadron production in central nucleus-nucleus collisions at chemical freeze-out," Nuclear Physics A, vol. 772, no. 3-4, pp. 167-199, 2006.

[6] C. Tsallis, "Possible generalization of Boltzmann-Gibbs statistics," Journal of Statistical Physics, vol. 52, no. 1-2, pp. 479-487, 1988.

[7] T. S. Biro, G. Purcsel, and K. Urmossy, "Non-extensive approach to quark matter," The European Physical Journal A, vol. 40, pp. 325-340, 2009.

[8] W. M. Alberico and A. Lavagno, "Non-extensive statistical effects in high-energy collisions," European Physical Journal A, vol. 40, no. 3, pp. 313-323, 2009.

[9] T. S. Biro and G. Purcsel, "Non-extensive Boltzmann equation and hadronization," Physical Review Letters, vol. 95, Article ID 162302, 2005.

[10] K. Urmossy, "Multiplicity dependence of Hadron spectra in proton-proton collisions at LHC energies and super-statistics," http://arxiv.org/abs/1212.0260.

[11] A. Adare, S. Afanasiev, C. Aidala et al., "Measurement of neutral mesons in $p+p$ collisions at $\sqrt{s}=200 \mathrm{GeV}$ and scaling properties of hadron production," Physical Review D, vol. 83, Article ID 052004, 2011.

[12] P. K. Khandai, P. Sett, P. Shukla, and V. Singh, "Hadron spectra in $\mathrm{p}+\mathrm{p}$ collisions at rhic and LHC energies," International Journal of Modern Physics A, vol. 28, no. 16, Article ID 1350066, 2013.

[13] M. Rybczynski and Z. Wlodarczyk, "Tsallis statistics approach to the transverse momentum distributions in p-p collisions," The European Physical Journal C, vol. 74, p. 2785, 2014.

[14] M. D. Azmi and J. Cleymans, "Transverse momentum distributions in proton-proton collisions at LHC energies and Tsallis thermodynamics," Journal of Physics G, vol. 41, Article ID 065001, 2014.

[15] J. Cleymans, G. I. Lykasov, A. S. Parvan, A. S. Sorin, O. V. Teryaev, and D. Worku, "Systematic properties of the tsallis distribution: energy dependence of parameters in high energy p-p collisions," Physics Letters B, vol. 723, no. 4-5, pp. 351-354, 2013.

[16] C. Y. Wong and G. Wilk, "Tsallis fits to $p_{T}$ spectra and multiple hard scattering in $p p$ collisions at the LHC," Physical Review D, vol. 87, Article ID 114007, 2013.

[17] R. Hagedorn, "Multiplicities, pT distributions and the expected hadron to quark-gluon phase transition," La Rivista del Nuovo Cimento, vol. 6, no. 10, pp. 1-50, 1984.

[18] R. Blankenbecler and S. J. Brodsky, "Unified description of inclusive and exclusive reactions at all momentum transfers," Physical Review D, vol. 10, no. 9, pp. 2973-2992, 1974.

[19] N. Abgrall, A. Aduszkiewicz, Y. Ali et al., "Measurement of negatively charged pion spectra in inelastic $\mathrm{p}+\mathrm{p}$ interactions at $p_{\text {lab }}=20,31,40,80$ and $158 \mathrm{GeV} / \mathrm{c}$," The European Physical Journal C, vol. 74, p. 2794, 2014.

[20] A. Adare, S. Afanasiev, C. Aidala (PHENIX Collaboration) et al., "Identified charged hadron production in $p+p \sqrt{s}=200$ and 62.4 GeV," Physical Review C, vol. 83, Article ID 064903, 2011.
[21] S. Chatrchyan, V. Khachatryan, A. M. Sirunyan et al., "Study of the inclusive production of charged pions, kaons, and protons in pp collisions at $\sqrt{s}=0.9,2.76,7 \mathrm{TeV}$," The European Physical Journal C, vol. 72, article 2164, 2012.

[22] R. Hagedorn, "Statistical thermodynamics of strong interactions at high energies," Nuovo Cimento, supplement 3, pp. 147186, 1965.

[23] D. B. Walton and J. Rafelski, "Equilibrium distribution of heavy quarks in Fokker-Planck dynamics," Physical Review Letters, vol. 84 , p. 31, 2000.

[24] G. Wilk and Z. Wlodarczyk, "Interpretation of the nonextensivity parameter $q$ in some applications of Tsallis statistics and Lévy distributions," Physical Review Letters, vol. 84, p. 2770, 2000.

[25] B. I. Abelev, J. Adams, M. M. Aggarwal et al., "Strange particle production in $p+p$ collisions at $\sqrt{s}=200 \mathrm{GeV}$," Physical Review C, vol. 75, Article ID 064901, 2007.

[26] J. Cleymans and D. Worku, "Relativistic thermodynamics: transverse momentum distributions in high-energy physics," The European Physical Journal A, vol. 48, article 160, 2012.

[27] R. Blankenbecler, S. J. Brodsky, and J. Gunion, "Analysis of particle production at large transverse momentum," Physical Review D, vol. 12, no. 11, pp. 3469-3487, 1975.

[28] S. J. Brodsky, H. J. Pirner, and J. Raufeisen, "Scaling properties of high pT inclusive hadron production," Physics Letters B: Nuclear, Elementary Particle and High-Energy Physics, vol. 637, no. 1-2, pp. 58-63, 2006.

[29] T. S. Biro, K. Urmossy, and G. G. Barnafoldi, "Pion and kaon spectra from distributed mass quark matter," Journal of Physics G, vol. 35, no. 4, Article ID 044012, 2008.

[30] K. Aamodt, N. Abel, U. Abeysekara et al., "Production of pions, kaons and protons in pp collisions at $\sqrt{s}=900 \mathrm{GeV}$ with ALICE at the LHC", The European Physical Journal C, vol. 71, p. 1655, 2011.

[31] J. Adams, M. M. Aggarwal, Z. Ahammed et al., "Identified hadron spectra at large transverse momentum in $\mathrm{p}+\mathrm{p}$ and $\mathrm{d}+\mathrm{Au}$ collisions $\sqrt{s_{N N}}=200 \mathrm{GeV}$," Physics Letters B, vol. 637, no. 3, pp. $161-169,2006$. 

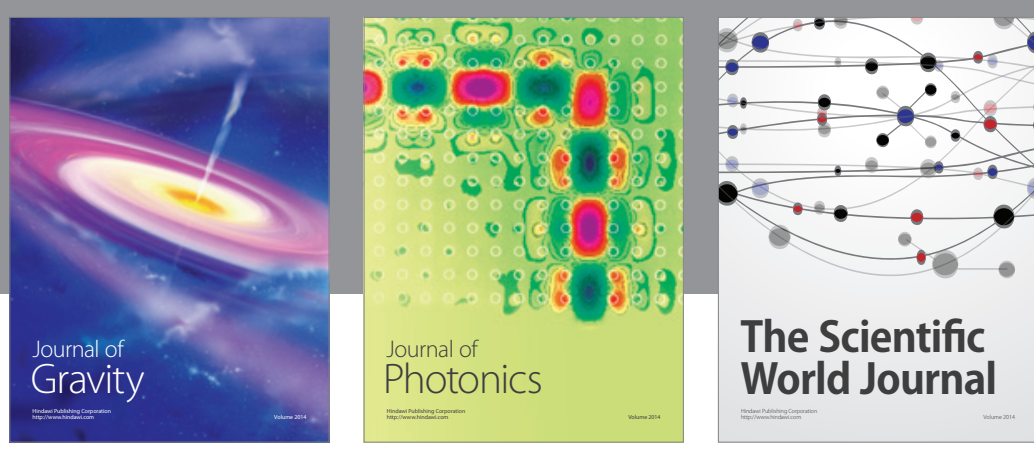

The Scientific World Journal
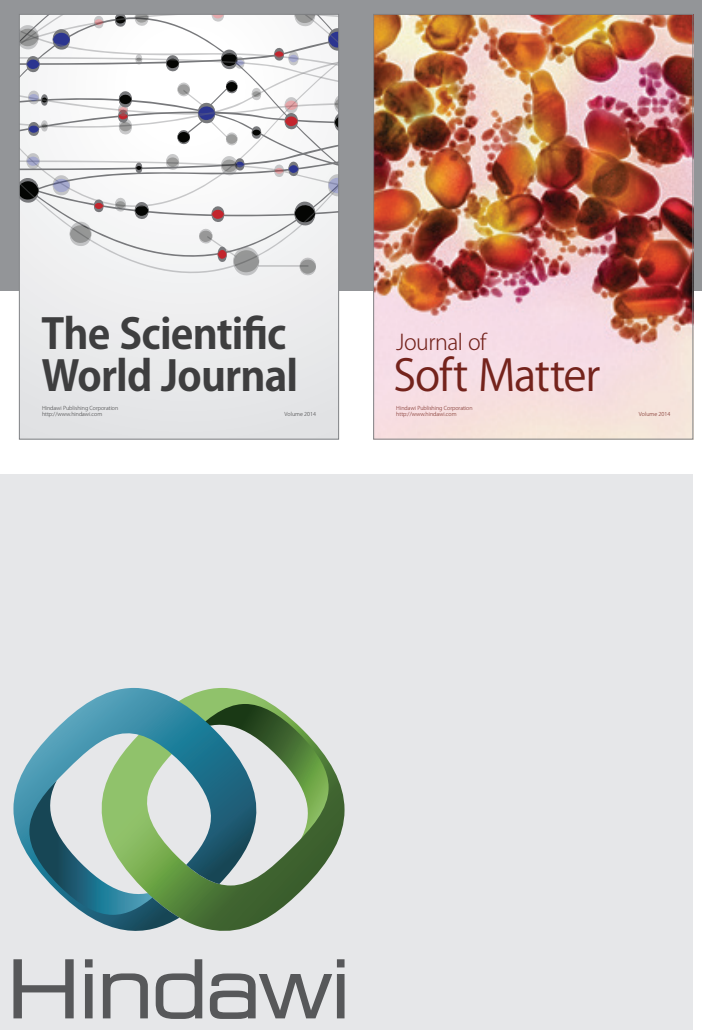

Submit your manuscripts at

http://www.hindawi.com

nternational Journal of

Statistical Mechanics
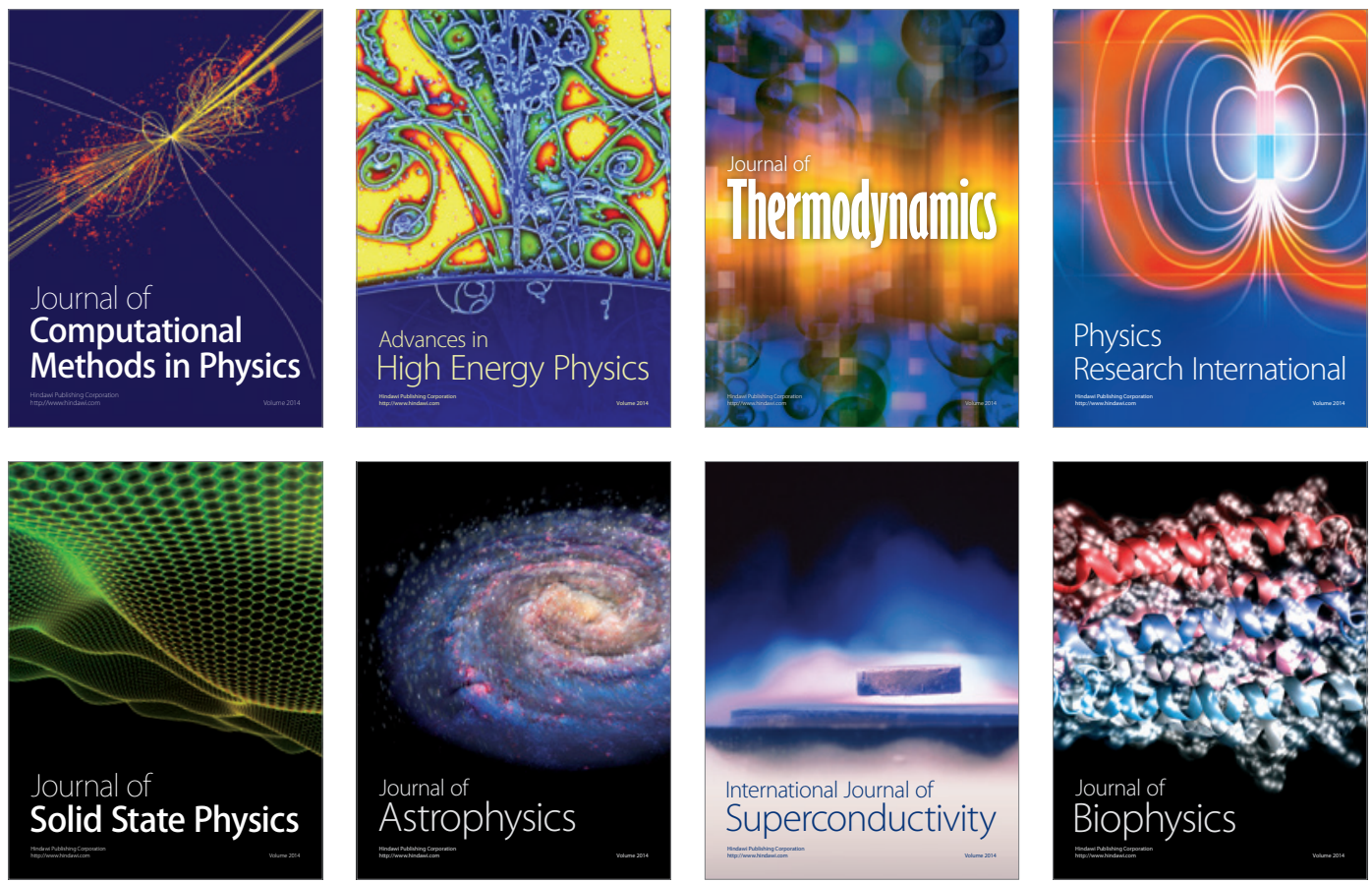
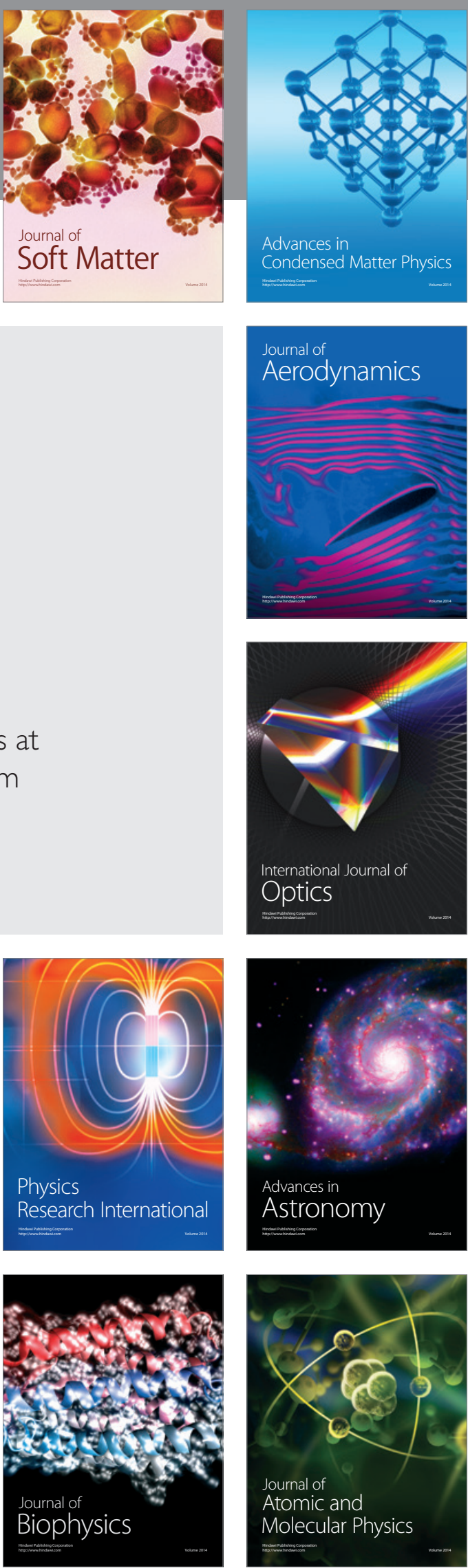